\title{
An alerting structure: human orexin receptor 1
}

\author{
Daniel Wacker \& Bryan L Roth \\ Structures of the human orexin receptor $1\left(\mathrm{hOX}_{1} \mathrm{R}\right)$ bound to a selective drug and the dual (hOX $\mathrm{R}$ - and $\mathrm{hOX}_{2} \mathrm{R}$ - \\ targeting) antagonist suvorexant reveal molecular mechanisms of selectivity in orexin-receptor subtypes.
}

Wakefulness in humans is mediated by activation of two orexinergic peptide receptors in the CNS, termed $\mathrm{hOX}_{1} \mathrm{R}$ and $\mathrm{hOX}_{2} \mathrm{R}^{1}$, which appear to 'kick-start' other neurotransmitter systems required for alertness ${ }^{2}$ and goaloriented behavior ${ }^{3}$. Not surprisingly, both orexin receptors are major targets for drug discovery to treat insomnia and other disorders of wakefulness ${ }^{4}$. In this issue, Yin et al. ${ }^{5}$ report the structure of $\mathrm{hOX}_{1} \mathrm{R}$ bound to the anti-insomnia drug suvorexant (Merck's Belsomra, the first US Food and Drug Administration-approved drug that targets both $\mathrm{hOX}_{1} \mathrm{R}$ and $\mathrm{hOX}_{2} \mathrm{R}$ ). The authors also report the structure of $\mathrm{hOX}_{1} \mathrm{R}$ in complex with the $\mathrm{hOX}_{1} \mathrm{R}$-selective ligand SB-647042; this structure, in combination with computational and functional studies, reveals the molecular mechanisms underlying orexinreceptor subtype selectivity ${ }^{5}$.

$\mathrm{hOX}_{1} \mathrm{R}$ and $\mathrm{hOX}_{2} \mathrm{R}$ are $\mathrm{G}$ protein-coupled receptors activated by two distinct peptides, orexin- $\mathrm{A}$ and orexin- $\mathrm{B}^{1,6}$, in the lateral hypothalamus, where they mediate the balance between wake- and sleep-promoting neuronal systems ${ }^{7}$. Activation of $\mathrm{hOX}_{1} \mathrm{R}$ and $\mathrm{hOX}_{2} \mathrm{R}$ promotes wakefulness, arousal and motivation via excitatory effects on many other neurotransmitter systems including serotonergic, dopaminergic, noradrenergic, and histaminergic

Daniel Wacker and Bryan L. Roth are at the Department of Pharmacology, University of North Carolina at Chapel Hill, Chapel Hill, North Carolina, USA. Bryan L. Roth is also at the Division of Chemical Biology and Medicinal Chemistry, Eshelman School of Pharmacy, University of North Carolina at Chapel Hill, Chapel Hill, North Carolina, USA, and at the National Institute of Mental Health Psychoactive Drug Screening Program (NIMH PDSP), School of Medicine, University of North Carolina at Chapel Hill, Chapel Hill, North Carolina, USA. e-mail:bryan_roth@med.unc.edu neurons ${ }^{7}$. Orexin-receptor-knockout mice show increased sleepiness with shortened but intact waking bouts ${ }^{8}$, and studies have reported a $90 \%$ loss in orexinergic neurons in human narcolepsy ${ }^{9}$. Chemogenetic approaches have shown that complementation of orexinreceptor loss improves narcoleptic symptoms ${ }^{10}$, and excitation or suppression of orexin neurons increases or decreases wakefulness in mice $^{11}$, thus further highlighting the essential role of orexin-receptor signaling in maintaining normal sleep patterns. Because an estimated $\sim 50 \%$ of adults in the United States suffer from symptoms of insomnia at least few nights a week ${ }^{12}$, a better understanding of the molecular basis of sleep homeostasis through $\mathrm{hOX}_{1} \mathrm{R}$ and $\mathrm{hOX}_{2} \mathrm{R}$ is of high therapeutic relevance.

Having previously determined the $\mathrm{hOX}_{2} \mathrm{R}$ structure $^{13}$, Yin et al. ${ }^{5}$ now present the first
$\mathrm{X}$-ray structure of $\mathrm{hOX}_{1} \mathrm{R}$, in the form of two cocrystal structures: (i) $\mathrm{hOX}_{1} \mathrm{R}$ bound to suvorexant and (ii) $\mathrm{hOX}_{1} \mathrm{R}$ bound to the $\mathrm{hOX}_{1} \mathrm{R}$-selective compound SB-674042. By combining the structural information with computational, functional and radioligand binding studies, Yin et al. ${ }^{5}$ provide unprecedented molecular insight into the ligand recognition and subtype selectivity of these orexinergic antagonists.

The hOX $\mathrm{R}_{1}$ structure reveals an N-terminal helix that is folded over the top of the ligandbinding site of the receptor (Fig. 1) and appears to be critically involved in recruiting and binding the orexin peptide for receptor activation ${ }^{5}$. This N-terminal helix is not visible in the previously published $\mathrm{hOX}_{2} \mathrm{R}$ crystal structure, but sequence conservation suggests that both subtypes possess this structural motif. Similarly to the $\mathrm{hOX}_{2} \mathrm{R}$ structure, suvorexant

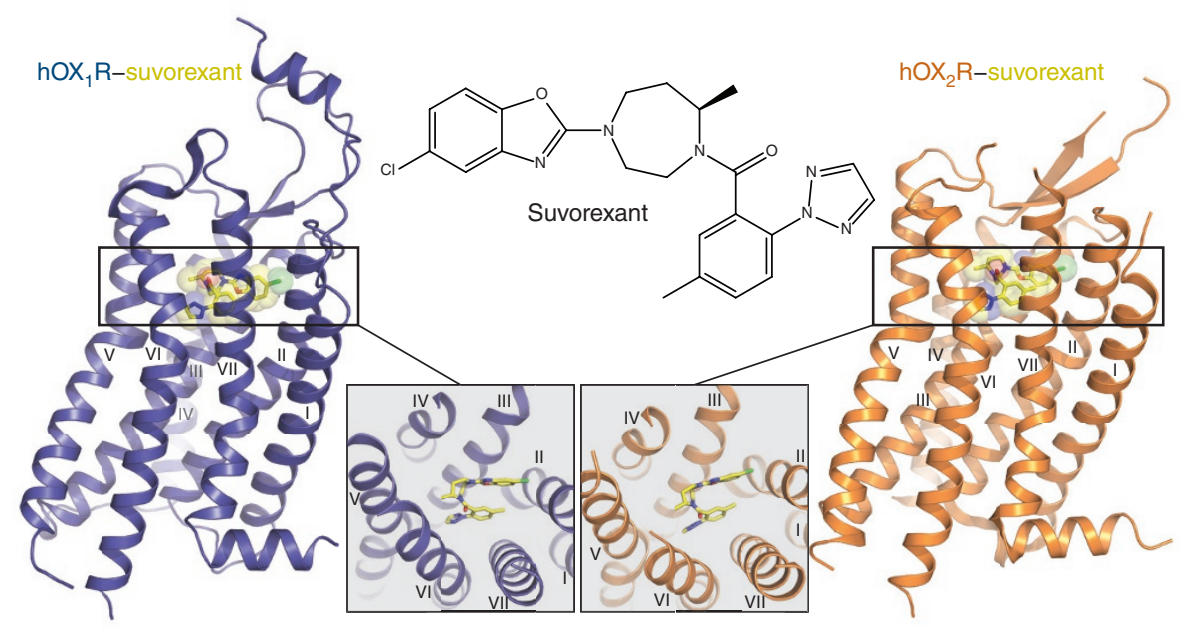

Figure 1 Crystal structures of the human orexin receptor 1 (hOX ${ }_{1}$, PDB $\left.4 Z J 8\right)^{5}$ and human orexin receptor $2\left(\mathrm{hOX}_{2} \mathrm{R}, \mathrm{PDB} 4 \mathrm{SOV}\right)^{13}$ bound to the anti-insomnia drug suvorexant. Seven transmembrane bundles of hOX $\mathrm{R}$ (blue) and $\mathrm{hOX}_{2} \mathrm{R}$ (orange) are shown with suvorexant (yellow) bound to the orthosteric site of the receptors. Insets show views of the orthosteric receptor sites from the extracellular space; extracellular residues have been removed for clarity. 
a

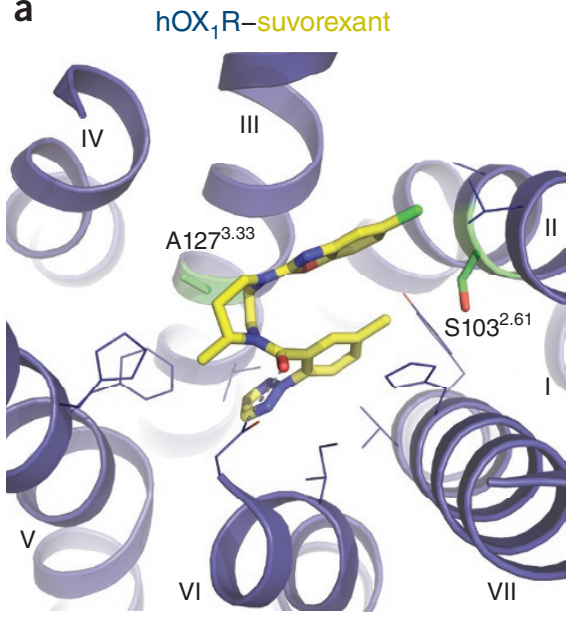

b

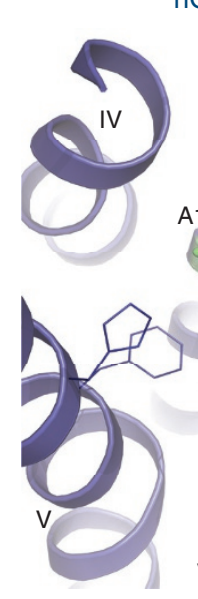

hOX $_{1}$ R-SB-674042

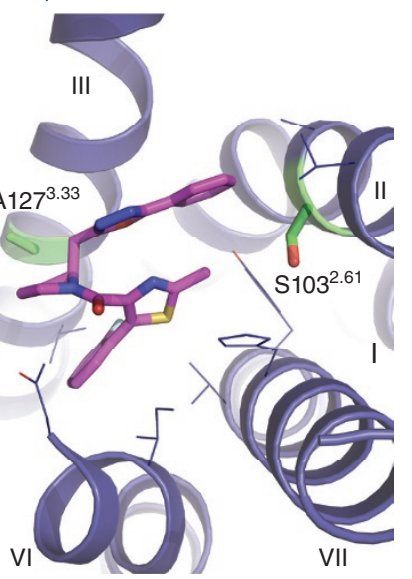

C

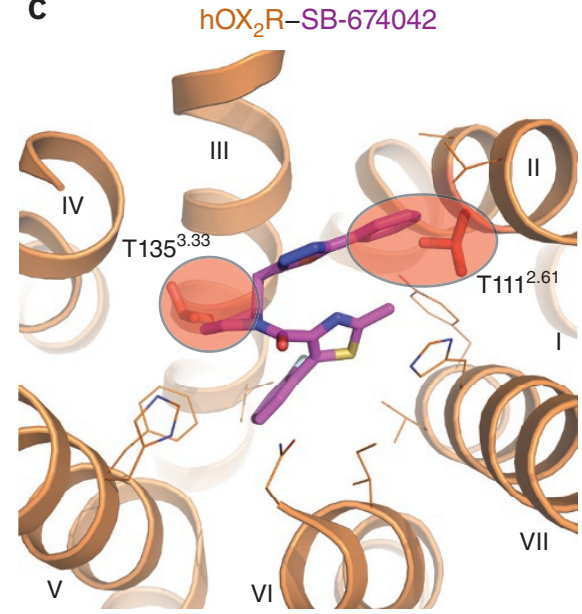

Figure 2 Comparison of the orthosteric binding sites of hOX ${ }_{1} \mathrm{R}$ and hOX 2 R. Orthosteric binding sites are shown from the extracellular space. Extracellular residues have been removed for clarity. (a,b) Crystal structures of hOX $\mathrm{R}$ (blue) with bound suvorexant (yellow) (a) and SB-674042 (magenta) (b).

(c) hOX $\mathrm{R}$-selective ligand SB-674042 docked into the binding site of the hOX $\mathrm{R}_{2}$ crystal structure (orange). hOX $\mathrm{R}^{-}$-receptor residues $\mathrm{A}_{12} 7^{3.33}$ and $\mathrm{S} 103^{2.61}$ (superscripts indicate Ballesteros-Weinstein numbering), which are responsible for binding the selective ligand SB-674042, are shown in green. hOX $_{2}$ R-receptor residues $T 135^{3.33}$ and $T 111^{2.61}$, which impair high-affinity binding of the hOX $\mathrm{R}_{1}$-selective ligand SB-674042 to hOX $\mathrm{R}_{2}$, as identified by docking and mutational analysis, are shown in red.

displays a horseshoe-like conformation in the binding pockets of both receptors (Fig. 1), although clear differences in the orientation of the compound are evident between receptor subtypes.

The $\mathrm{hOX}_{1} \mathrm{R}$-selective SB-674042 compound reveals a conformation nearly identical to that of suvorexant, despite their completely different chemotypes (Fig. 2). From a drug-optimization perspective, it is interesting to note that the ligand-receptor contacts are predominantly hydrophobic, although both ligands contain numerous polar sites capable of forming of hydrogen bonds. To investigate ligand selectivity at the orexin receptors, the authors performed docking experiments of the $\mathrm{hOX}_{1} \mathrm{R}$-selective SB-674042 compound into the binding site of the $\mathrm{hOX}_{2} \mathrm{R}$ structure and identified likely unfavorable contacts with two threonine residues. Indeed, the authors elegantly showed that mutating those threonines to the corresponding $\mathrm{hOX}_{1} \mathrm{R}$ residues markedly increases the affinity of the hOX $\mathrm{X}_{1} \mathrm{R}$-selective compound but does not affect the binding of nonselective compounds.

Elucidating the molecular mechanisms of subtype selectivity at these important therapeutic targets represents a considerable advance, given the high sequence homology and similar pharmacology of $\mathrm{hOX}_{1} \mathrm{R}$ and $\mathrm{hOX}_{2} \mathrm{R}$. These similarities have made it challenging to develop suitable chemical tools to study the distinct roles of each receptor in human physiology. Despite their restriction to the lateral hypothalamus, the orexin system projects to and receives inputs from several areas of the brain that mediate motivation and anxiety ${ }^{2,7}$, including the limbic system and amygdala. It is thus conceivable that some projections may be governed by both orexin receptors, whereas others may be controlled by a single receptor subtype. Consequently, the development of orexin-receptor ligands with increased subtype selectivity may not only facilitate the study of distinct orexinergic pathways but also provide a path toward therapeutic intervention in conditions associated with a specific subtype. In fact, the $\mathrm{hOX}_{2} \mathrm{R}$-selective antagonist LSN2424100 shows antidepressant effects in rodents, whereas the $\mathrm{hOX}_{1} \mathrm{R}$ selective antagonists SB334867 and ACT-335827 appear to reduce fear and compulsive behaviors ${ }^{14-16}$.

These studies highlight the therapeutic promise of orexin-targeted drugs beyond their application in sleep disorders and, importantly, underscore the distinct roles of $\mathrm{hOX}_{1} \mathrm{R}$ and $\mathrm{hOX}_{2} \mathrm{R}$ in human physiology. Given the recent advances in structure-based virtual ligand screening ${ }^{17}$ and rational drug design ${ }^{18}$, the molecular insights into orexin-receptor function presented by Yin et al. ${ }^{5}$ should greatly facilitate the generation of new chemical matter, providing a promising structural foundation for new orexinergic therapeutics for a variety of pathological conditions.

\section{COMPETING FINANCIAL INTERESTS}

The authors declare no competing financial interests.

1. Sakurai, T. et al. Cell 92, 573-585 (1998).

2. Li, J., Hu, Z. \& de Lecea, L. Br. J. Pharmacol. 171 332-350 (2014).

3. Boutrel, B., Cannella, N. \& de Lecea, L. Brain Res. 1314, 103-111 (2010)

4. Roecker, A.J., Cox, C.D. \& Coleman, P.J. J. Med. Chem 59, 504-530 (2016).

5. Yin, J. et al. Nat. Struct. Mol. Biol. 23, 293-299 (2016).

6. de Lecea, L. et al. Proc. Natl. Acad. Sci. USA 95 322-327 (1998).

7. Kumar, A., Chanana, P. \& Choudhary, S. Pharmacol. Rep. 68, 231-242 (2016)

8. Diniz Behn, C.G., Klerman, E.B., Mochizuki, T. Lin, S.C. \& Scammell, T.E. Sleep 33, 297-306 (2010).

9. Thannickal, T.C. et al. Neuron 27, 469-474 (2000).

10. Hasegawa, E., Yanagisawa, M., Sakurai, T. \& Mieda, M. J. Clin. Invest. 124, 604-616 (2014).

11. Sasaki, K. et al. PLoS One 6, e20360 (2011).

12. Gershell, L. Nat. Rev. Drug Discov. 5, 15-16 (2006).

13. Yin, J., Mobarec, J.C., Kolb, P. \& Rosenbaum, D.M. Nature 519, 247-250 (2015).

14. Steiner, M.A. et al. ChemMedChem 8, 898-903 (2013).

15. Flores, Á. et al. Neuropsychopharmacology 39 2732-2741 (2014)

16. Fitch, T.E. et al. Front. Neurosci. 8, 5 (2014).

17. Kolb, P. et al. Proc. Natl. Acad. Sci. USA 106, 6843-6848 (2009).

18. Congreve, M. et al. J. Med. Chem. 55, 1898-1903 (2012). 